\title{
30 Die Revolution 1905 und ihre Folgen auf der Krim
}

\begin{abstract}
Nach der Hinrichtung bitte ich, mit Nachdruck und allen Mitteln, dass mein Leichnam vor den Arbeitern Sewastopol's aufgebahrt wird, damit sie Herr und Meister der Beerdigung sind. Ich bin ihr Vertreter, ich bin stolz auf diesen Titel, denn sie [die Arbeiter Sevstopol's, KSJ] gaben meinem Leben mehr Glück, als all die Leute, die je ich getroffen habe. [...] Wenn die Stadt irgendwann ein Monument errichten wird, dann sollen sie in den Stein meinen Eid eingravieren. [...] Ich erhob das Banner der Revolution der russischen Flotte, die dem Volk treu blieb, und lasst diese Flagge der Freiheit auf meinem Grab flattern. ${ }^{1}$
\end{abstract}

Der Verfasser dieser Zeilen war Leutnant Petr P. Šmidt (1867-1906), der im Verlauf der Russischen Revolution von 1905 eine Reihe revolutionärer, gegen die zarische Regierung gerichteter Aktivitäten entfaltete; er schrieb sie kurz vor seiner Hinrichtung. Nach mehreren Zusammenstößen mit der Ordnungsmacht und daraus resultierenden Verhaftungen hatte er im Herbst 1905 auf dem in Sevastopol' vor Anker liegenden Panzerkreuzer „Očakov“ das Kommando über die aufständischen Matrosen übernommen. Das Hissen der Roten Fahne gelang, nicht aber die geplante Einnahme strategisch wichtiger Punkte der Stadt und die Verhaftung der kaisertreuen Offiziere. In einem von Šmidt unterzeichneten Telegramm war Zar Nikolaus II. (1868-1918) zur sofortigen Einberufung einer verfassungsgebenden Versammlung aufgefordert worden. Das hatte allerdings keine Folgen. Regierungstreuen Einheiten gelang eine rasche Niederschlagung der Revolte. Der sich als unabhängiger Sozialist bezeichnende Šmidt war zwar nicht der Einzige, aber der bekannteste unter den Verhafteten in Sevastopol', das während der ersten Russischen Revolution der zentrale Kriegshafen der zarischen Marine im Schwarzen Meer war. Nach einem kurzen Gerichtsverfahren unter Ausschluss der Öffentlichkeit wurden er und einige seiner Mitstreiter zum Tode verurteilt und auf der kleinen, heute unbewohnten Insel Berezan' in der Nähe des Dnepr-Bug-Limans hingerichtet. Im Abschiedsbrief des bis heute in den Nachfolgestaaten der UdSSR populären Helden des „revolutionären Kampfes der Volksmassen“, wie es in Publikationen aus sowjetischer Zeit hie $\beta,{ }^{2}$ hatte er nicht nur den Wunsch nach einer öffentlichen Trauerfeier geäußert, sondern wollte zudem seinen Leichnam an der Seite anderer Opfer der revolutionären Ereignisse von 1905 in Sevastopol' beerdigt wissen. St. Petersburg erfüllte dieses Ansinnen - wenig erstaunlich nicht. Erst nach einem weiteren revolutionären Ereignis, der Februarrevolution

1 Pis'mo (1983), 107.

2 Vgl. z.B. Vaneev (1983), 12.

○ OpenAccess. ( 2020 Kerstin S. Jobst, publiziert von De Gruyter. ((c))/BY Dieses Werk ist lizenziert unter der Creative Commons Attribution 4.0 International. https://doi.org/10.1515/9783110520620-032 


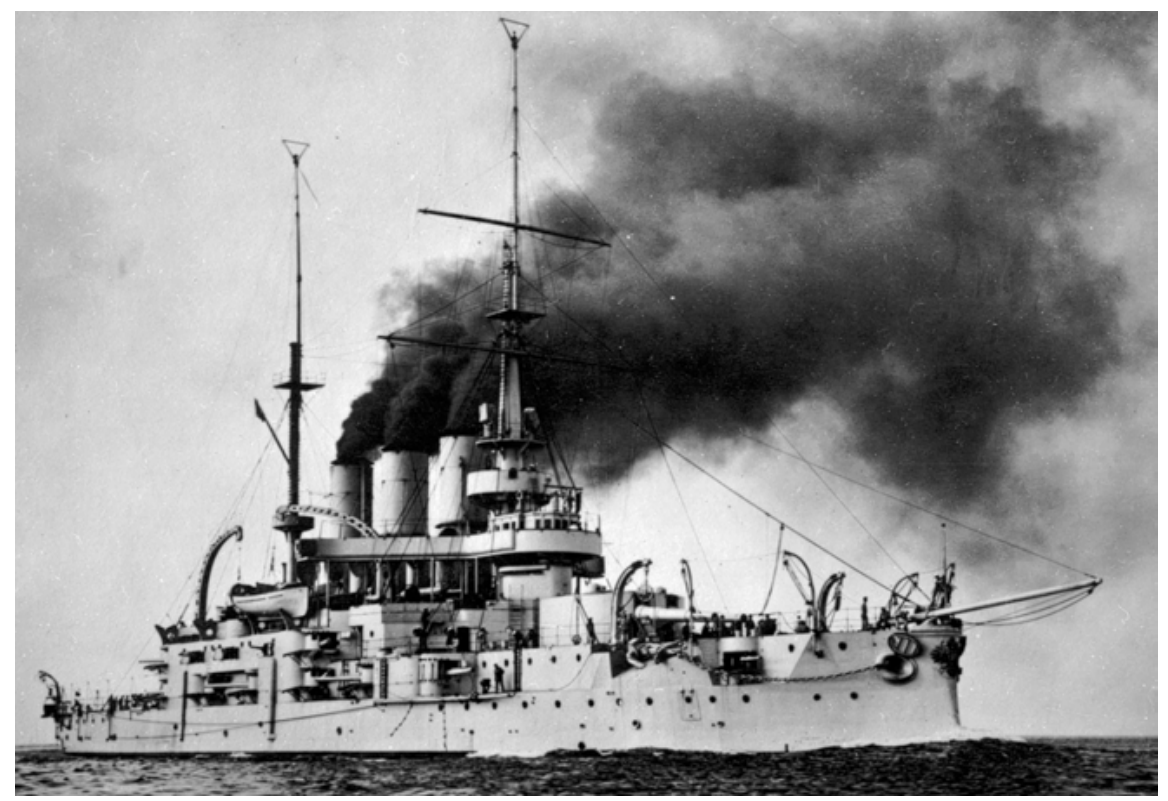

Abb. 11: Panzerschiff „Panteleimon“ (ehemals „Knjaz’ Potemkin-Tavričeskij“), Fotografie von 1906

von 1917, wurden Šmidts Überreste an seine revolutionäre Wirkstätte zurückgebracht. Dies geschah ausgerechnet unter dem sozialistischen Strömungen strikt abgeneigten damaligen Befehlshaber der Schwarzmeerflotte und späteren Protagonisten der sog. Weißen Bewegung, Admiral Aleksandr V. Kolčak (1874-1920). Um die aufrührerische Stimmung unter den Matrosen und Soldaten zu besänftigen, ließ er einige Leichname der Opfer von 1905, darunter auch den Šmidts, exhumieren und im Mai 1917 in der Sevastopol'er Pokrovskij-Kathedrale neu bestatten. Diese Geste half tatsächlich, die Lage vorerst zu beruhigen. 1923 fanden Šmidts Gebeine auf dem städtischen Friedhof Sevastopol's ihre letzte Ruhe. ${ }^{3}$ Ihren Sinn für Symbolträchtigkeit bewiesen die Bol’ševiki, als sie Šmidts Grabstein aus der Grabplatte des während der Meuterei auf dem Panzerkreuzer „Potemkin“ 1905 von Aufständischen getöteten Kapitäns Evgenij N. Golikov (1854-1905) fertigen ließen, der AnhängerInnen der zarischen Ordnung als positive Erinnerungsfigur galt. ${ }^{4}$

3 Smolin (2012), $165 \mathrm{f}$.

4 Vgl. als Beispiel für die positive Bewertung Golikovs: Tjuljakov (2014). 
Auch der Panzerkreuzer „Potemkin“ wurde zu einem revolutionären Symbol: Durch Sergej Ėjzenštejns (1898-1948) gleichnamigen Film (russ.: „Bronenosec Potëmkin“) von 1925, der nicht zuletzt wegen der berühmten „Treppenszene“ eines der wohl am häufigsten zitierten Werke der Filmgeschichte ist, ${ }^{5}$ wird außerhalb des russischsprachigen Raumes die Revolution von 1905 vor allem mit den Ereignissen auf der „Potemkin“ in Odessa assoziiert. Allerdings blieb die Revolution keineswegs auf St. Petersburg und Moskau bzw. Odessa beschränkt. ${ }^{6}$ Sowohl auf dem Land als auch in den nicht mehrheitlich russisch besiedelten Peripherien des Imperiums wie im Baltikum, der Kaukasusregion oder in Polen kam es zu revolutionären Ausschreitungen. ${ }^{7}$ So auch auf der Krim. Die Begebenheiten des Jahres 1905 trugen dazu bei, dass Sevastopol' in der russischen Erinnerungskultur als ein militärisch-revolutionärer Ort eingeschrieben ist, ${ }^{8}$ auch wenn der Krimkrieg bzw. der Krieg von 1939/41-1945 ohne Zweifel noch stärker kollektiv erinnert werden. Die sowjetische Geschichtspolitik nutzte Leutnant Šmidt und seine Mitstreiter, um der Vorstellung von Sevastopol' als exponiertem revolutionären hot spot Nachdruck zu verleihen. Zudem versuchte die sowjetische Geschichtsschreibung, eine aufständisch-revolutionäre Traditionslinie zu konstruieren, die schon vor 1905 ihren Anfang genommen haben soll: So wurde beispielsweise der durch Lebensmittelknappheit verursachte sog. ženskij oder čumnoj bunt (Frauen- bzw. Pestaufstand) von 1830 immer wieder als Beginn der revolutionären Geschichte der Stadt interpretiert. ${ }^{9}$ Leutnant Šmidt schließlich, obzwar kein Bol'ševik, war Teil der die ganze Sowjetunion - vom westlichen Tver' bis zum östlichen Vladivostok ${ }^{10}$ - durchziehenden revolutionär-heroischen materiellen und immateriellen Denkmal- und Erinnerungskultur: Brücken, wie etwa die erste feste Brücke über die Neva in Leningrad (St. Petersburg), die seit 2007 Blagoveščenskij-Brücke heißt, wurden nach ihm benannt; zahlreiche AutorInnen nahmen sich seiner an, unter ihnen Boris L. Pasternak (1890-1960), Autor des

5 Nachzuschauen unter folgendem Link: http://cinema.arte.tv/de/artikel/die-treppenszene-auspanzerkreuzer-potemkin (Stand 14.03.2018).

6 Zum Forschungsstand der Revolution von 1905 vgl. vor allem Kusber (2007). Vgl. auch Ascher (1988/1994).

$7 \mathrm{Zu}$ den verschiedenen revolutionären Schauplätzen vgl. den Sammelband Frings u. Kusber (2007) sowie z.B. Tych (1990).

8 Vgl. dazu die eher für LiebhaberInnen der Militärgeschichte geeignete Publikation von Melvin (2017).

9 Vgl. z.B. Polkanov (1936). Vgl. auch Jobst (2017b), 166.

10 Im russischen Wikipedia-Eintrag zu Šmidt sind z. B. - ohne Anspruch auf Vollständigkeit mehr als fünfzig Städte in der ehemaligen Sowjetunion genannt, in denen ihm mittels einer Straßenbenennung, eines Denkmals oder einer Erinnerungstafel gedacht worden war: Šmidt (2018). Vgl. auch Qualls (2009), 138. 
„Doktor Živago“, der ihm ein Poem widmete, und es gibt mindestens zwei Opern, die nach ihm heißen - eine von Nikolaj I. Platonov (1894-1967) aus dem Jahr 1938, eine andere von B. L. Jarovinskij (1922-2000) von 1970.

Aufruhr - und aus der Perspektive der jeweiligen Machthaber sind Revolutionen und Aufstände nichts anderes als ein Aufstand gegen eine als legitim angesehene Ordnung - ist in einer so stark militärisch geprägten Stadt wie Sevastopol' nicht ungewöhnlich: Die Bevölkerung von Hafenstädten gilt allgemein als „turbulent und unkontrollierbar.“11 Wie verlief das Jahr 1905 aber in den anderen Teilen der Krim? Wie stand es mit der Teilnahme bäuerlicher und intellektueller Tataren und Tatarinnen - eine Industriearbeiterschaft im klassischen Sinne gab es auf der agrarisch geprägten Halbinsel nur in Ansätzen - und anderer nationaler Gruppen? In städtischen, gemischt besiedelten Gebieten außerhalb Sevastopol's wie in Feodosija oder Simferopol' kam es vereinzelt zu Streiks und 1905 auch zu Demonstrationen zum 1. Mai, während in den von TatarInnen bewohnten Dörfern kein besonderer Aufruhr festzustellen war. ${ }^{12} \mathrm{Ab}$ Ende des Jahres 1905 zeigte sich aber, dass die revolutionären Ereignisse zu einer bis dahin unvorstellbaren „Organisierung der Gesellschaft“ beitrugen ${ }^{13}$ : In Regie örtlicher Akteure, weitgehend ohne die Intervention zugereister Agitatoren, formierten sich bäuerliche Organisationen. Vereinzelt kam es zu Landarbeiterstreiks, und tatarische Kleinbauern, die traditionell mit slavischen und muslimischen Grundbesitzern über ihre traditionellen Landnutzungsprivilegien stritten, fühlten sich ermuntert, ihre Rechte beherzter einzufordern. ${ }^{14}$ Wie in anderen Teilen des Imperiums kam es im Verlauf der Ereignisse auch zu antisemitischen Ausschreitungen, welche zum Teil von den staatlichen Stellen vor Ort initiiert worden waren. ${ }^{15}$

Die imperiumsweite Bewegung der RusslandmuslimInnen, die ja durch Gasprralı und den Djadidismus (Kapitel 29) seit den 1880er Jahren wesentliche Impulse bekommen hatte, erfuhr durch das Jahr 1905 weiteren Antrieb. Bald sollte sich zeigen, dass Kazan' oder Orenburg zu den eigentlichen Zentren einer muslimischen Bewegung wurden. Auf der Krim begannen dessen ungeachtet unter den muslimischen Eliten bis dahin unbekannte Debatten über das Wesen krimtatarischer Identität abseits von Religion und Sprache. Diese hatten auch einen

11 So Osterhammel (2009), 402.

12 Vozgrin (1992), 362.

13 Neutatz (2013), 111.

14 Ganz ähnlich verhielten sich tatarische Bauern im Wolga-Gebiet, vgl. Noack (2000), 235.

15 Vozgrin (1992), 363f., betont, dass sich Krimtataren nicht an antisemitischen Ausschreitungen beteiligt hätten. In einem Fall sei eine berittene tatarische Einheit zur Auflösung der „Pogromščiki“ (Pogromtäter) eingesetzt worden. 
generationellen Aspekt: Während ältere, etablierte Akteure sich mit bildungspolitischen Erfolgen wie der Gründung von über 350 Schulen mit tatarischer und russischer Sprache auf der Halbinsel begnügten, ${ }^{16}$ stellten Jüngere weitergehende Fragen nach dem Verhältnis der KrimtatarInnen zu anderen RusslandmuslimInnen, dem Russländischen Imperium und dem Osmanischen Reich. Waren KrimtatarInnen eine von den übrigen RusslandtatarInnen bzw. von den TürkInnen distinkte Gruppe? War einer spezifisch krimtatarischen Sprachvariante der Vorzug zu geben oder einer anderen? Wie sollten die ökonomischen und politischen Probleme der krimtatarischen BewohnerInnen gelöst werden - innerhalb des Zarenreichs, in Anbindung an Istanbul oder unabhängig von beiden Mächten? ${ }^{17}$ Und welche Ausprägung hatten die vielgestaltigen pantürkischen Elemente der Debatten ${ }^{18}$ Es zeigte sich, dass es in diesen intellektuellen Diskursen nicht mehr primär um die Bedeutung des Islam als identitätsstiftendes Moment ging, wie es noch einige Dekaden davor der Fall gewesen war und wie es ja grundsätzlich auch noch auf Gaspıralı zutraf, der in der Religion einen wichtigen Orientierungspunkt sah. ${ }^{19}$ In der neben Bağçasaray wichtigsten tatarischen Stadt auf der Krim, in Qarasuvbazar, sammelten sich die Akteure einer häufig als Jungtataren (Genç Tatarlar) bezeichneten Gruppe, welche die ,krimtatarische Frage‘ sozial, politisch und eben auch national definierten. Sie äußerten Zweifel, ob das autokratische russländische System diese zu lösen im Stande sei. Zentrale Figur war der spätere Bürgermeister Qarasuvbazars, Abdurreşit Mehdi (in russischer Form auch Rešid Medievič Mediev, 1880-1912), der Abgeordneter in der nur zwischen Februar und Juni 1907 tagenden und dann aufgelösten zweiten Duma war. In dieser waren 36 der 450 Abgeordneten Muslime, die ursprünglich eine eigene Fraktion gebildet hatten, von der sich aber einige aus dem Kaukasus und dem Wolga-Gebiet stammende Abgeordnete wegen abweichender Auffassungen in der Agrarfrage abspalteten. ${ }^{20}$ In Wahlbezirken, in denen keine eigenen Kandidaten aufgestellt werden konnten, hatte man mit der liberalen Partei der Konstitutionellen Demokraten („Kadetten“) kooperiert. In der russländischen Volksvertretung, die dem Zaren durch den imperiumsweiten Druck geradezu abgerungen worden war, dominierten politisch links verortete Politiker, was ein Grund für ihre Kurzlebigkeit war. ${ }^{21}$ Auch Mehdi stand politisch sozialrevolutionären Positionen nah. Be-

16 Fisher (1978), 104.

17 Magocsi (2014), $75 \mathrm{f}$.

18 Pekesen (2014).

19 Lazzerini (1997), 177.

20 Dazu im Detail Noack (2000), $256 \mathrm{f}$.

21 Zudem wurde der Modus zu den Wahlen der 3. Duma zu Lasten nationaler Minderheiten verändert. 
sonders die Not der landarmen krimtatarischen Bauern wurde sein Thema, forderte er doch die Verbesserung von deren Lage durch die Verteilung des in den Händen der Gutsbesitzer, des Staates oder der muslimischen Gemeinschaft befindlichen Landes. ${ }^{22}$ Trotz seiner vergleichsweise radikalen Ansichten wurde er einer der stellvertretenden Sprecher der muslimischen Duma-Fraktion, die sich ansonsten programmatisch an den Kadetten orientierten. ${ }^{23}$ In der Rückschau spielt Mehdi in der Geschichte der krimtatarischen nationalen Identitätsbildung vor allen Dingen deshalb eine Rolle, da er wohl als erster die Krim als Entität sui generis definiert hat: Sie war für ihn „not a province of the Russian Empire, segment of the Dar al-Islam [Haus des Islams, d.h. alle Gebiete unter muslimischer Herrschaft] or adjunct of a larger Turkish homeland, but as the national patrimony of the Crimean nation. “24 Obgleich er und seine Anhänger die etablierte muslimische Geistlichkeit als Hauptgegner ansahen, bediente er sich einer religiös gefärbten Sprache, um die krimtatarische Bauernschaft zu erreichen. Dies war aber nur eine sprachliche Anpassung, denn letztlich ist die Taktik dieser Bewegung mit jener der frühen russischen Sozialisten der Narodniki (Volkstümler oder Volksfreunde) zu vergleichen, sowohl in ihrer antireligiösen Haltung als auch in ihrem Antimonarchismus. ${ }^{25}$

Dieser Entwicklung vorausgegangen war ein erster, von muslimischen Delegierten aus allen Teilen des Imperiums beschickter Muslim-Kongress im August 1905 in Nižnij Novgorod, dem 1906 ein zweiter in St. Petersburg folgen sollte. Fragen der Verbesserung des Bildungswesens sowie rechtlicher, juristischer und religiöser Probleme der RusslandmuslimInnen wurden diskutiert. Gaspiralı konnte seine Vorstellungen von der Notwendigkeit einer Einbindung in und Orientierung am russländischen System vorerst noch erfolgreich einbringen. ${ }^{26}$ Man einigte sich in Erwartung der Installierung einer Staatsduma auf die Gründung einer muslimischen Partei. Im Endergebnis kam es vorerst ,nur` zur Gründung des „Bundes der Muslime Russlands“ (Rusiya Musulmannarynyng Ittifaky bzw. Rusiya Musulmanlarinın İttifakı, kurz: Ittifak). Ittifak befand sich immerhin, so Christian Noack, ,auf dem Weg zur Partei.“27 In dieser wurden jedoch alsbald, genauso wie in der eher als Einheit gedachten denn real existierenden Gruppe der RusslandmuslimInnen insgesamt, die Unterschiede evident. Auf der Krim gab es neben den Anhängern Gaspiralıs und den sog. Jungtataren um Mehdi und ihrem

22 Williams (2001), 320.

23 Noack (2000), 258.

24 Williams (2001), 320.

25 Williams (2001), $321 \mathrm{f}$.

26 Fisher (1978), 104.

27 Noack (2000), 254. 
Journal „Vatan Hadimi“ („Diener der Nation“) bald eine dritte, mal als „Nationalisten“ (Fisher), mal als „Separatisten“ (Vozgrin) bezeichnete Gruppe. ${ }^{28}$ In jedem Fall war diese Strömung zeittypisch, formierten sich doch in den Jahrzehnten vor dem Ersten Weltkrieg allenthalben nationale Bewegungen, die ihr definiertes ,nationales Schicksal‘ außerhalb der großen Imperien zu erfüllen glaubten.

Die Köpfe dieser Bewegung unter den Krimtataren waren der Jurist und spätere Mufti Noman Çelebicihan (1885-1918) und Cafer Seydahmet Qırımer (1889-1960), der ebenfalls ein Studium der Rechtswissenschaft abgeschlossen hatte; beide sollten während der revolutionären Ereignisse auf der Krim in den Jahren 1917 und 1918 noch wichtige Rollen spielen (Kapitel 31). ${ }^{29}$ Beide lernten sich vermutlich um das Jahr 1908 in Istanbul kennen, wo sie wie viele andere RusslandmuslimInnen auch ihre Ausbildung komplettierten. ${ }^{30}$ Die Erfahrung der sog. Jungtürkischen Revolution in dieser Zeit mag zur im Osmanischen Reich erfolgten Politisierung der beiden Akteure und der übrigen krimtatarischen Diaspora beigetragen haben. Durch die Wiedereinführung der Verfassung eröffneten sich im Osmanischen Reich auch für RusslandmuslimInnen einschließlich der KrimtatarInnen neue Freiheiten, die sich u.a. in journalistischen Aktivitäten niederschlugen. Sie gründeten zudem verschiedene teils geheim, teils offen agierende politische Vereine. Als Ziel wurde schließlich die ,Befreiung der krimtatarischen Nation' definiert. ${ }^{31}$ Eine von Seydahmet in der osmanischen Hauptstadt verfasste Broschüre mit dem Titel „Die unterdrückte krimtatarischen Nation im 20. Jahrhundert“ führte 1911 sogar zur diplomatischen Intervention St. Petersburgs, welches von den osmanischen Behörden seine Verhaftung forderte. Dieser entzog er sich aber durch seine Flucht nach Paris. ${ }^{32}$ Ohnehin beobachteten russisch-imperiale Stellen mit großer Aufmerksamkeit die Tätigkeiten ihrer muslimischen UntertanInnen im Osmanischen Reich, sahen sie darin doch potentielle Probleme sowohl für die innerimperialen als auch für die außenpolitischen Konstellationen. ${ }^{33} 1909$ hatte sich in Istanbul unter Seydahmets und Çelebicihans Ägide die „Vatan-Gesellschaft“ (Vatan Cemiyeti) formiert, die in der Forschung übereinstimmend als wichtige Etappe in der nationalpolitischen Genese einer sich an das Territorium der Halbinsel bindenden krimtatarischen Identität bewertet wird: „The Members of the Fatherland Society were proposing nothing less than independent Crimean Tatar statehood in their Crimean home-

28 Fisher (1978), 106; Vozgrin (1992), 381.

29 Vgl. beide Kurzbiographien bei Kogonašvili (1995), 252 und 310.

30 Dazu grundlegend Adam (2002).

31 Dazu ausführlicher Kirimli S. (1990), 102-104.

32 Bowman (2005).

33 Vgl. Adam (2002), 194f. sowie 428-448. 
land“, so beispielsweise Williams. ${ }^{34}$ Den im Exil politisierten krimtatarischen Rückkehrern gelang der Aufbau eines zum Teil illegalen Kommunikationsnetzes, das ,nationales' Schriftgut verteilte, Lesezirkel gründete und Multiplikatoren wie Lehrer in den Gaspıralıschen Reformschulen in ihrem Sinne beeinflussen konnte. Von einem die Mehrheit der krimtatarischen Bevölkerung durchdringenden Nationalbewusstsein kann allerdings zu diesem Zeitpunkt nicht die Rede sein; auch hier ist der krimtatarische Fall vergleichbar mit dem anderer nichtdominierender Nationalitäten in den osteuropäischen Landimperien. Die mobilisierten Eliten konnten die Frage nach ihrem ultimativen Ziel - nach Unabhängigkeit oder einer stärker ausgeformten pantürkischen Verbindung unter den Tataren im Russländischen Reich oder in Anlehnung an das jungtürkische Beispiel - nicht final beantworteten. Nach 1905 zeigten sich einige Aktivisten enttäuscht über Gaspiralı. Dieser sei zu ,flexibel‘, lehnte revolutionäre Strömungen ab und sei vor allen Dingen ,gegenüber dem Zaren, der Reichsduma und der bestehenden Gesellschaftsordnung“ zu loyal. Der Umstand, dass er „das Vertrauen des Innenministeriums genoß“, machte ihn verdächtig. ${ }^{35}$ Der Nachwuchs verfolgte weitergehende Ziele, deren Erreichung spätestens ab 1917 möglich erschien. Dies erlebte der trotz aller Kritik auch heute noch von Musliminnen und Muslimen hochgeschätzte Gaspiralı nicht mehr, verstarb er doch im September 1914 nach kurzer und schwerer Krankheit.

34 Williams (2001), 325.

35 Adam (2002), 94. 